University of New Hampshire

University of New Hampshire Scholars' Repository

$8-1-2008$

\title{
Metrics for solar wind prediction models: Comparison of empirical, hybrid, and physics-based schemes with 8 years of L1 observations
}

\author{
M. J. Owens \\ Harlan E. Spence \\ Boston University, harlan.spence@unh.edu \\ S. McGregor \\ W. J. Hughes \\ J. M. Quinn
}

See next page for additional authors

Follow this and additional works at: https://scholars.unh.edu/physics_facpub

Part of the Physics Commons

\section{Recommended Citation}

Owens, M. J., H. E. Spence, S. McGregor, W. J. Hughes, J. M. Quinn, C. N. Arge, P. Riley, J. Linker, and D. Odstrcil (2008), Metrics for solar wind prediction models: Comparison of empirical, hybrid, and physicsbased schemes with 8 years of L1 observations, Space Weather, 6, S08001, doi:10.1029/2007SW000380.

This Article is brought to you for free and open access by the Physics at University of New Hampshire Scholars' Repository. It has been accepted for inclusion in Physics Scholarship by an authorized administrator of University of New Hampshire Scholars' Repository. For more information, please contact Scholarly.Communication@unh.edu. 


\section{Authors}

M. J. Owens, Harlan E. Spence, S. McGregor, W. J. Hughes, J. M. Quinn, C. N. Arge, P. Riley, J. Linker, and D. Odstrcil 


\title{
Metrics for solar wind prediction models: Comparison of empirical, hybrid, and physics-based schemes with 8 years of L1 observations
}

\author{
M. J. Owens, ${ }^{1}$ H. E. Spence, ${ }^{1}$ S. McGregor, ${ }^{1}$ W. J. Hughes, ${ }^{1}$ J. M. Quinn, ${ }^{1}$ C. N. Arge, ${ }^{2}$ \\ P. Riley, ${ }^{3}$ J. Linker, ${ }^{3}$ and D. Odstrcil ${ }^{4}$ \\ Received 10 December 2007; revised 20 May 2008; accepted 21 May 2008; published 7 August 2008.
}

[1] Space weather effects on technological systems originate with energy carried from the Sun to the terrestrial environment by the solar wind. In this study, we present results of modeling of solar coronaheliosphere processes to predict solar wind conditions at the L1 Lagrangian point upstream of Earth. In particular we calculate performance metrics for (1) empirical, (2) hybrid empirical/physics-based, and (3) full physics-based coupled corona-heliosphere models over an 8-year period (1995-2002). L1 measurements of the radial solar wind speed are the primary basis for validation of the coronal and heliosphere models studied, though other solar wind parameters are also considered. The models are from the Center for Integrated Space-Weather Modeling (CISM) which has developed a coupled model of the whole Sun-to-Earth system, from the solar photosphere to the terrestrial thermosphere. Simple point-bypoint analysis techniques, such as mean-square-error and correlation coefficients, indicate that the empirical coronal-heliosphere model currently gives the best forecast of solar wind speed at $1 \mathrm{AU}$. A more detailed analysis shows that errors in the physics-based models are predominately the result of small timing offsets to solar wind structures and that the large-scale features of the solar wind are actually well modeled. We suggest that additional "tuning" of the coupling between the coronal and heliosphere models could lead to a significant improvement of their accuracy. Furthermore, we note that the physicsbased models accurately capture dynamic effects at solar wind stream interaction regions, such as magnetic field compression, flow deflection, and density buildup, which the empirical scheme cannot.

Citation: Owens, M. J., H. E. Spence, S. McGregor, W. J. Hughes, J. M. Quinn, C. N. Arge, P. Riley, J. Linker, and D. Odstrcil (2008), Metrics for solar wind prediction models: Comparison of empirical, hybrid, and physics-based schemes with 8 years of L1 observations, Space Weather, 6, S08001, doi:10.1029/2007SW000380.

\section{Introduction}

[2] Prediction of the solar wind in near-Earth space is highly desirable due to the variety of adverse effects "space weather" can have on both ground- and spacebased technologies [e.g., Feynman and Gabriel, 2000]. While the majority of large geomagnetic storms are driven by interplanetary coronal mass ejections (ICMEs) [e.g., Tsurutani et al., 1988], the ambient solar wind conditions

\footnotetext{
${ }^{1}$ Center for Space Physics, Boston University, Boston, Massachusetts, USA.

${ }^{2}$ Space Vehicles Directorate, Air Force Research Laboratory, Kirtland Air Force Base, New Mexico, USA.

${ }^{3}$ Science Applications International Corporation, San Diego, California, USA.

${ }^{4}$ Cooperative Institute for Research in Environmental Sciences, University of Colorado, Boulder, Colorado, USA.
}

are important both geoeffectively in their own right [e.g., Richardson et al., 2002] and for modulating the arrival time and geoeffectiveness of ICMEs.

[3] The Center for Integrated Space-Weather Modeling (CISM) is a NSF/Science Technology Center (STC) that aims to couple together physics-based numerical models of the whole Sun-Earth system, from the solar photosphere to the terrestrial thermosphere, for both scientific and forecast applications [Luhmann et al., 2004]. In order for these coupled simulations to be a useful tool for the community, it is imperative to assess and track the predictive capabilities of the component and coupled models throughout their development, as was done for atmospheric weather models through the 20th century [e.g., Siscoe et al., 2004]. 
[4] This paper aims to quantitatively assess model performance in the prediction of ambient solar wind conditions in near-Earth space. We note that the coronal models derive solar wind speed from quasi-empirical relations to the coronal magnetic field configuration (see sections 2.1 and 2.2), meaning such a measure of performance may not necessarily be representative of how well the coronal field topology has been reconstructed. Independent tests, such as comparison between model open flux regions and observed coronal holes [e.g., de Toma et al., 2005], are required for such purposes.

[5] The standard method for assessing the quality of a prediction is to calculate the correlation or the meansquare-error (MSE) between the observed and model time series. If the observed (model) value of a parameter at time $t$ is denoted $X_{t}\left(X_{t}^{\prime}\right)$, then the MSE of $N$ observations is given by:

$$
M S E=\frac{1}{N} \sum_{t=1}^{N}\left(X_{t}-X_{t}^{\prime}\right)^{2}
$$

[6] While useful, such metrics do not differentiate between periods that are difficult to predict and periods when the model simply performs poorly (e.g., in comparison to other models). For this reason, it is desirable to compare a model's accuracy with the equivalent predictions of an unchanging "baseline" model, so as to compute the relative "skill" of the model under test [e.g., Siscoe et al., 2004; Spence et al., 2004]. Thus the skill of the model being tested can be defined as

$$
\text { Skill }=100\left(1-\frac{M S E}{M S E_{R E F}}\right)
$$

where MSE is the mean square error between the model and observations, and $M S E_{R E F}$ is the corresponding value for the baseline model. $M S E_{R E F}$ will be unchanging in time, and thus the skill of the model under test should increase as improvements and refinements are made. Note that the skill of a model can range from +100 to $-\infty$.

\section{Component Models}

[7] For both computational and physical reasons, the corona and heliosphere are best modeled separately. The various component models are described in this section.

\subsection{WSA Model of the Corona}

[8] The Wang-Sheeley-Arge model (WSA) [Arge and Pizzo, 2000; Arge et al., 2003] is based upon a modified potential field source surface (PFSS) model [Schatten et al., 1969; Altschuler and Newkirk, 1969] of the steady-state corona. Photospheric magnetic field maps are used to create Carrington maps of radial photospheric field, which serve as input to the PFSS to determine the coronal field out to 2.5 solar radii $\left(2.5 R_{S}\right)$. The output of the PFSS model serves as input to the Schatten Current Sheet (SCS) model [Schatten, 1971], which provides a more realistic magnetic field topology of the upper corona. The following empirical relationship (similar in form to Arge et al. [2003] and Owens et al. [2005]) is used to assign solar wind speed at a radius of $5 R_{S}$ :

$$
\begin{aligned}
V\left(f_{s}, \theta_{b}\right)= & 265+\frac{1.5}{\left(1+f_{s}\right)^{1 / 3}} \\
& \cdot\left\{5.8-4.4 \exp \left[1-\left(\theta_{b} / 7.5^{\circ}\right)^{3}\right]\right\}^{3.5} \mathrm{~km} \mathrm{~s}^{-1}
\end{aligned}
$$

It is a function of two coronal parameters, flux tube expansion factor $\left(f_{s}\right)$, and the minimum angular separation (at the photosphere) between an open field foot point and its nearest coronal hole boundary $\left(\theta_{b}\right.$, measured in degrees). This relationship differs from Arge and Pizzo [2000], which was based only on expansion factor:

$$
V\left(f_{s}\right)=267.5+\frac{410}{f_{s}^{2 / 5}} \mathrm{~km} \mathrm{~s}^{-1}
$$

The addition of the $\theta_{b}$ parameter was in part influenced by the success of MHD models that used this parameter [e.g., Riley et al., 2001], as described below.

\subsection{MAS Model of the Corona}

[9] The global corona is also modeled by the Magnetohydrodynamics Around a Sphere (MAS) 3-D MHD code developed by the SAIC group [Linker et al., 1999; Mikic et al., 1999] (see also http://www.imhd.net). Photospheric magnetic field observations provide the boundary conditions, from which initial conditions are derived by a potential field solution to the photospheric radial field, a uniform boundary density and a Parker-type solar wind outflow. The time-dependent polytropic MHD equations, with finite resistivity and viscosity, are then solved in spherical geometry between 1 and $30 R_{S}$ in a nonrotating frame. The solution is allowed to relax to steady state. See Linker and Mikić [1997] for a description of the initial and boundary conditions and Lionello et al. [1999] for details of the algorithm. For the 8 years of model results required by this metric study, we used a low-resolution nonuniform mesh with 60, 70, and 64 grid cells in the radial, meridional, and azimuthal directions, respectively.

[10] The MHD solutions used in this paper employ a polytropic energy equation. This approach avoids the the complicated physics of the transition region (e.g., radiative loss, anisotropic thermal conduction, and coronal heating) by solving an ideal energy equation and setting the ratio of specific heats $(\gamma)$ to a reduced value $(\gamma=1.05$ for the solutions shown). The polytropic approach yields nearly isothermal solutions, which qualitatively reproduce many coronal properties such as coronal hole and streamer morphology. However, the plasma velocity does not reproduce the large speed variation that is observed between the fast and slow solar wind. To provide lower 
boundary conditions for the heliospheric MHD model (see section 2.4), the plasma velocity from the coronal model is replaced with an empirically derived velocity:

$$
V_{r}\left(\theta_{b}\right)=V_{\text {slow }}+\frac{1}{2}\left(V_{\text {fast }}-V_{\text {slow }}\right)\left[1+\tanh \left(\frac{\theta_{b}-\alpha}{w}\right)\right],
$$

where $\theta_{b}$ is the minimum distance from an open-closed boundary, measured along the surface of the photosphere, $\alpha$ is a measure of how thick the slow flow band is $\left(\sim 0.05\right.$ radians, or $\left.\sim 2.86^{\circ}\right)$, and $w$ is the width over which the flow is raised to coronal hole values $(\sim 0.025$ $\mathrm{rad}$, or $\sim 1.43^{\circ}$ ). $V_{\text {slow }}=250 \mathrm{~km} / \mathrm{s}$, and $V_{\text {fast }}=650 \mathrm{~km} / \mathrm{s}$. $V_{r}\left(\theta_{b}\right)$ is computed at the photosphere from a map of the open-closed boundaries generated from the MHD solution. This quantity is then mapped outward along field lines to generate $V_{R}$ at the inner boundary condition of the heliospheric model (usually at 20$\left.30 R_{S}\right)$. It is not used in the coronal part of the solution.

[11] This expression was developed from the observation that in mapping studies, the slow wind typically maps back to the boundaries of coronal holes, while the fast wind arises from deeper within coronal holes [Linker et al., 1999]. The only coronal parameter the solar wind speed depends on is $\theta_{b}$ (see Riley et al. [2001] for further details). While the solar wind specification given above does not depend explicitly on expansion factor, the magnetic field near coronal hole boundaries typically has a large expansion factor, so the specification does have some correspondence with the WSA model. In general, the implications of deriving solar wind speed from coronal field topology (i.e., equations (3) and (5)) as the size, shape, and location of coronal holes vary over the solar cycle [Harvey and Recely, 2002] have yet to be fully explored.

\subsection{Kinematic Model of the Heliosphere}

[12] The simplest method we use to propagate the nearSun solar wind (as derived by the coronal models) to $1 \mathrm{AU}$ is a 1-D modified kinematic code which uses an ad hoc method to account for stream interactions (see Arge and Pizzo [2000] and Arge et al. [2004] for more details). This technique is regularly used in conjunction with the WSA coronal model to forecast solar wind speed and magnetic field polarity in near-Earth space [Arge et al., 2003].

[13] We extend the standard WSA predictions to infer additional solar wind properties in the same manner as Owens et al. [2005]: nonradial components of the solar wind flow are assumed to be zero. Proton density is estimated by assuming constant mass flux, and proton temperature is derived from the speed-temperature relation of Lopez [1987]. To calculate a magnetic field, the vector is assumed to lie in the ecliptic plane with the model solar wind speed used to compute a Parker spiral angle and assuming an intensity of $5 \mathrm{nT}$. This gives a complete set of basic solar wind magnetic field and plasma parameters without requiring modification of the existing models. We note, however, that the open flux computed by the PFSS model is not used by the baseline model.

\subsection{ENLIL Model of the Heliosphere}

[14] The heliosphere can also be modeled by the "ENLIL" 3-D ideal MHD code developed at NOAA/SEC [e.g., Odstrcil, 2003, and references therein]. The computational domain covers $21.5 \mathrm{R}_{S}$ to $1 \mathrm{AU}$ and $-60^{\circ}$ to $+60^{\circ}$ in solar latitude.

[15] The lower boundary conditions for the ENLIL model are provided by a coronal solution (either MAS or WSA), with the radial component of the magnetic field $\left(B_{R}\right)$ used directly. The radial plasma velocity is $\left(V_{R}\right)$ is provided by either equation (3) for the WSA model or by equation (5) for the MAS model.

[16] The meridional component of the magnetic field $(B \theta)$ is assumed to be zero, whereas the azimuthal component $\left(B_{\varphi}\right)$ is derived from the rotation speed of the source surface (i.e., $B_{\varphi}=-B_{R}\left(V_{R O T} / V_{R}\right) \sin \theta$, where $V_{R O T}$ is derived from the 27.2753 day rotation period of the Sun). The nonradial components of the plasma flow are assumed to be zero on the ENLIL inner boundary. Density and temperature are specified from the assumptions of constant momentum flux and thermal pressure balance along a flux tube, respectively. The heliospheric solution is allowed to relax to steady state.

[17] Again, as 8 years of model results are required, we use the low-resolution version of the ENLIL code for computational efficiency. It uses 128, 30, and 90 grid cells in the radial, meridional, and azimuthal directions, respectively.

\section{Coupled Models}

[18] The first routinely available in situ observations along the solar-terrestrial chain are of solar wind just upstream of Earth, meaning coronal and heliospheric models are often considered in tandem for the purpose of validation. This section describes the configuration of the coupled models used in this study. Figure 1 shows a summary of the constituent components of the coupled models. Note that in this study the models are all used to calculate a steady-state solar wind, potentially allowing prediction of ambient solar wind conditions, but not transient features such as ICMEs.

\subsection{Baseline Model}

[19] The CISM "baseline" model is the WSA coronal model (section 2.1), using photospheric magnetic field maps from the National Solar Observatory (NSO) at Kitt Peak (http://synoptic.nso.edu) at $5^{\circ}$ spatial resolution, propagated from $5 R_{S}$ to $1 \mathrm{AU}$ using the kinematic method described in section 2.3. This solar wind prediction scheme is in regular use at the Space Weather Prediction Center (http://www.swpc.noaa.gov/ws/). 


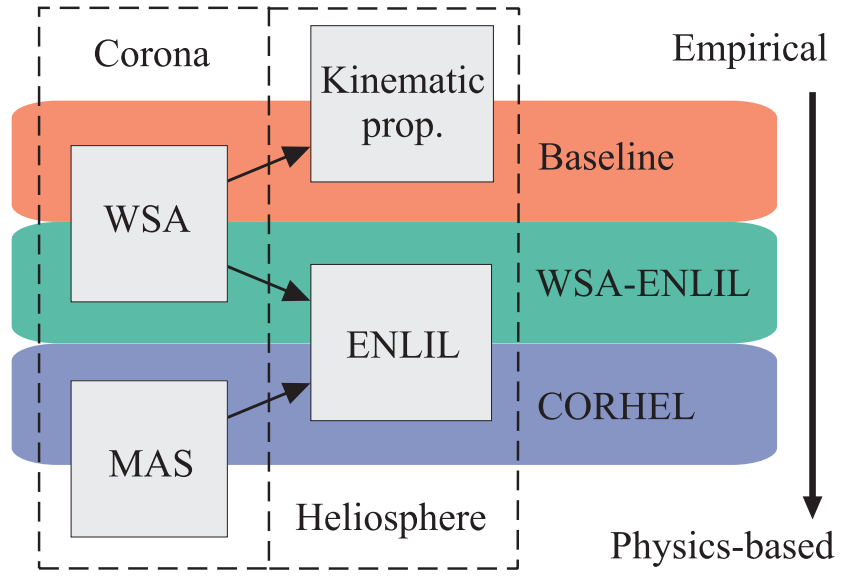

Figure 1. A schematic representation of the constituent components that go into the coupled models tested in this study.

\subsection{WSA-ENLIL}

[20] The WSA empirical coronal model (section 2.1) can also be used to initiate the ENLIL MHD model of the heliosphere (section 2.4), yielding a hybrid empirical/ physics-based coupled model. The version of WSA-ENLIL used in this study is Version 1.0, which is based upon WSA driven by NSO synoptic maps. The WSA derived solar wind speed is ballistically propagated from the WSA corona outer boundary at $5 R_{S}$ to the ENLIL inner boundary at $21.5 R_{S,}$.

\subsection{CORHEL}

[21] One of the goals of CISM is to provide coupled physics-based models of the whole Sun-Earth system. To this end, the "CORHEL" model couples the MAS MHD model of the corona (section 2.2) with the ENLIL MHD model of the heliosphere (section 2.4). In this study, MAS is driven with magnetograms from NSO and is coupled to ENLIL at $30 R_{S}$. See Odstrcil et al. [2004] and Luhmann et al. [2004] for more detail. The low-resolution mode of CORHEL version 2.3 is used in this study.

\section{Results}

[22] We compare the model-predicted time series with observations of the solar wind upstream of the Earth. All time series are averaged to an $1-\mathrm{h}$ resolution. The models all run in heliographic coordinates, but we convert model time series at L1 into geocentric-solar-ecliptic (GSE) coordinates for comparison with spacecraft data.

[23] It is instructive to begin with a detailed analysis of the solar wind speed, the main prediction of the baseline model, before considering the other solar wind parameters. We note that when periods of solar wind identified as ICMEs are removed from the time series, the overall metrics are not significantly altered (not shown). As the bulk properties of the solar wind have been shown to be primarily determined by ambient flows rather than transients [Richardson et al., 2002], this is not surprising.

\subsection{Solar Wind Speed Metrics}

[24] Figures 2, 3, and 4 show the baseline, WSA-ENLIL, and CORHEL-predicted radial solar wind speeds at L1, respectively. The black plots are the observed values from the Wind (1995-1997) and ACE (1998-2002) spacecraft. Any data gaps, resulting from missing data at L1 or incomplete magnetograms to initiate the models, occurring in any of the time series have been removed from all four time series to ensure equal coverage. By eye, all three model time series track the observed large-scale features of the solar wind reasonably well.

[25] Table 1 shows the various statistics of the model and observed solar wind time series. As can also be seen directly from the time series plots, the baseline model displays a similar mean and variability in solar wind speed to that observed, resulting in a low MSE. The baseline model has clearly been well-tuned to the prediction of the solar wind at $1 \mathrm{AU}$, an advantage of using a reasonably mature empirical model. The WSA-ENLIL model also matches the mean solar wind speed very well, but the variability in solar wind speed is much lower than observed. This is particularly apparent during 1995 and 1996. Conversely, CORHEL matches the solar wind variability well, but the mean speed is too low. This offset to the mean results in a higher MSE (and hence lower skill) than the WSA-ENLIL model, despite better tracking the overall structure of the solar wind speed. The offset to the CORHEL mean is mostly the result of the CORHEL slow wind speed during solar maximum being too low ( $\sim 50 \mathrm{~km} / \mathrm{s}$ slower than that observed. A simple tuning of the constants in equation (5) may be required). This demonstrates that MSE is not always a good measure of a model's predictive capability, particularly in the case of solar wind speed [Owens et al., 2005].

[26] Figure 5 shows the skill of CORHEL (blue) and WSA-ENLIL (green) in a 1-year rolling window. The accuracy of the CORHEL and WSA-ENLIL reconstructions of the solar wind speed are comparable to the baseline throughout 1995, 1996, and 1997. Both CORHEL and WSA-ENLIL show a drop-off in skill near the end of 1997, when the CME frequency and heliospheric current sheet inclination start to rapidly increase. From Figure 2, however, it can be seen that the skill drop is primarily due to the consistently low-speed solar wind, coupled with the baseline model performing particularly well, throughout this period. For WSA-ENLIL, this is just a temporary drop, and the skill returns to approximately zero (except during in 2002, due to the overprediction of the duration of highspeed streams). For CORHEL, however, the skill remains low through 1998-2002. This is due to the underprediction in the speed of the slow wind at this time. This is discussed in further detail in section 5 .

[27] Figure 6 shows the effect of introducing a systematic delay into the observed time series. The minimum RMS 


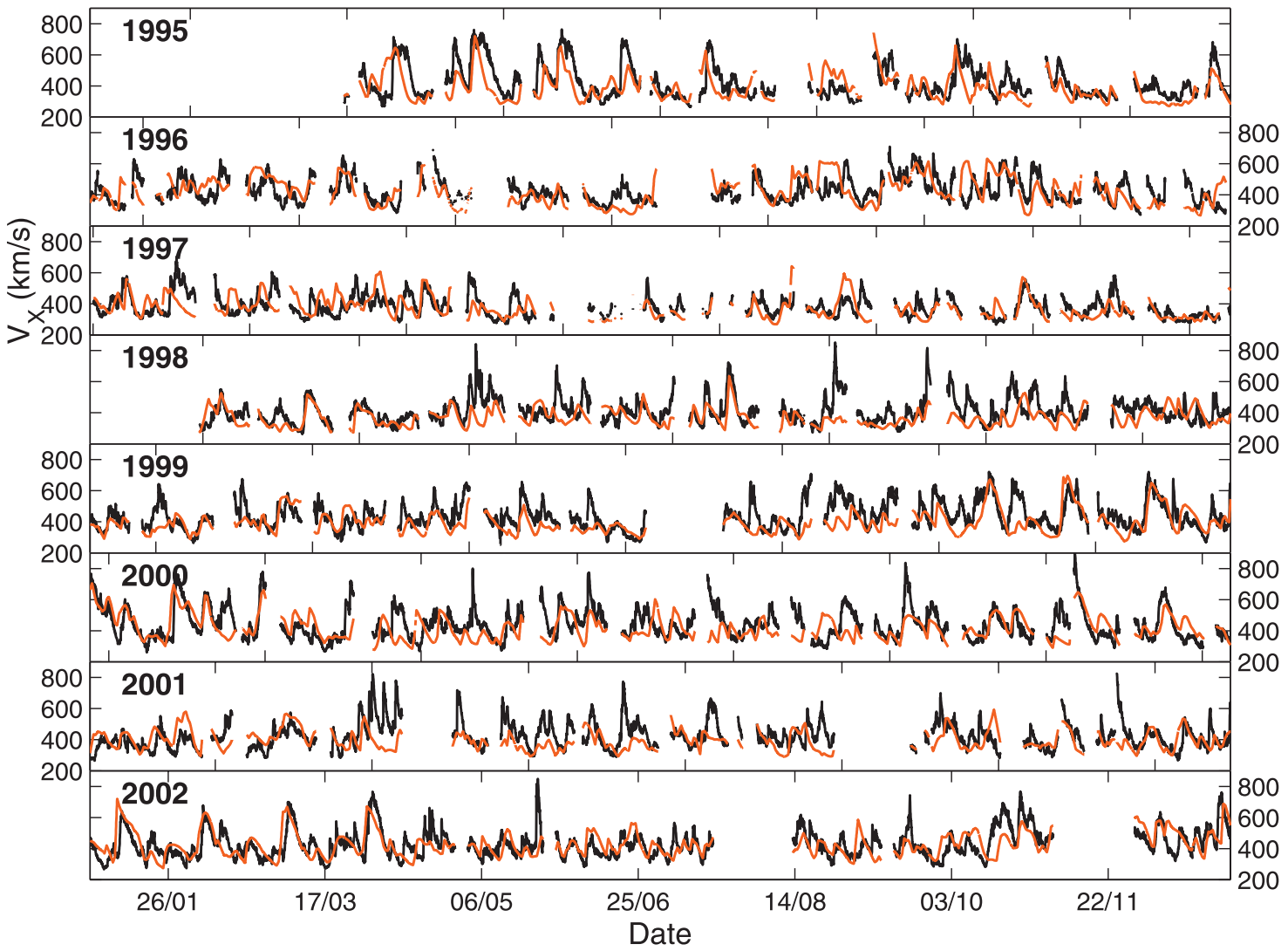

Figure 2. A comparison of the baseline prediction of the solar wind radial speed at L1 (red) with that observed (black) for the years 1995-2002. Both time series are at 1-h resolution.

error between the baseline prediction and observed solar wind speed occurs for approximately zero time offset, suggesting no significant time offset to the solar wind speed prediction. This is not surprising as the solar wind speeds inferred from equation (3) has been well tuned to match observations at 1 AU. For WSA-ENLIL and CORHEL, however, there is a systematic offset to the $V_{X}$ prediction, particularly at solar minimum (dashed lines, taken to be 1995, 1996, and 1997), with the model prediction being $\sim 2$ days early. This effect is less pronounced at solar maximum (dotted lines, take to be 1998 through 2002), when the RMS error is larger in general.

\subsection{High-Speed Enhancements}

[28] An event-based approach to model validation is often desirable, both for model developers and forecasters, as it gives insight into the strengths and weaknesses of models that point-by-point analysis (such as MSE and correlation based approaches) cannot. In particular, the effect of small timing errors and small, systematic offsets can be greatly reduced [e.g., Owens et al., 2005]. For solar wind speed, it is "high-speed enhancements" (HSEs) that both best define the accuracy of solar wind reconstruction and that are of most immediate interest to forecasters.
[29] As per Owens et al. [2005], we define a HSE as any region of the solar wind in which there is a net solar wind speed increase $\geq 100 \mathrm{~km} / \mathrm{s}$ in 2 days or less, and consider the number of hit, miss, and false HSE predictions in the baseline, WSA-ENLIL, and CORHEL time series. Table 2 summarizes the number of hit, miss, and falsely predicted HSEs by the three coupled models. A large number of missed HSEs are expected as fast ICMEs are not present in the models but are present in the observed time series. The ratio of hit to false HSEs is very favorable, from a forecast perspective, for all three coupled models. Note that WSA-ENLIL misses far more HSEs than CORHEL due predominantly to the reduced variability in the WSAENLIL solar wind speed during 1995-1996: Many of the WSA-ENLIL speed enhancements during this period fail to meet the criteria for a HSE and thus become "missed" events. Of course, the WSA-ENLIL underprediction of HSEs also results in even fewer "false" predictions.

[30] Figure 7 shows the distributions of the errors in the arrival time (top) and maximum speed (bottom) for the three models. The solid vertical lines show the mean of the distributions. As can also be seen from Table 3, all three models slightly underestimate the maximum speed of HSEs, particularly at solar maximum. As fast ejecta are frequently the cause of HSEs, and/or embedded in ambient 


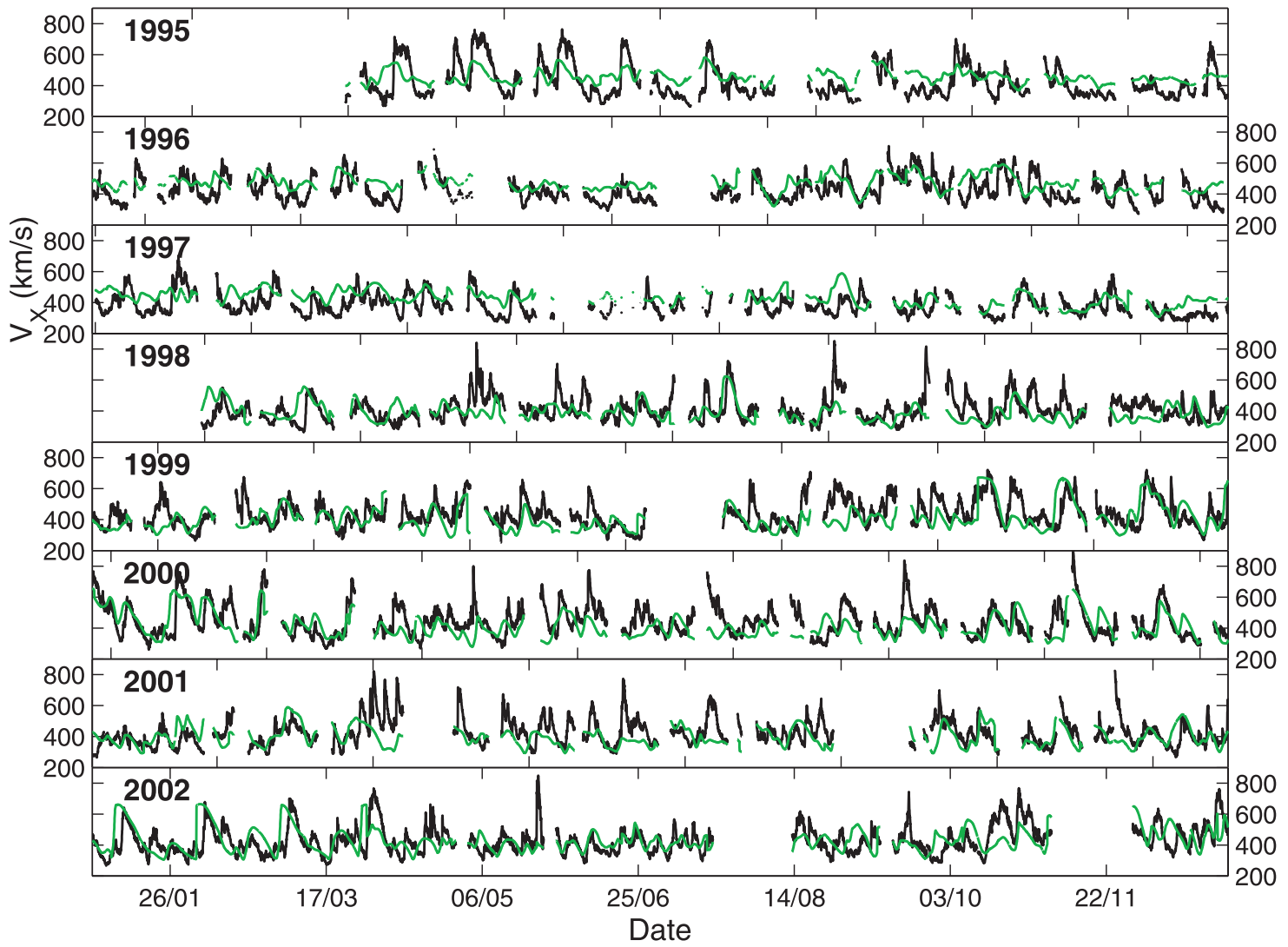

Figure 3. A comparison of the WSA-ENLIL prediction of the solar wind radial speed at L1 (green) with that observed (black) for the years 1995-2002. Both time series are at 1-h resolution.

solar wind HSEs, this result is not surprising. We also find an early arrival of WSA-ENLIL and CORHEL HSEs at solar minimum, which is less pronounced at solar maximum, as suggested by the RMS analysis shown in Figure 6. In general, CORHEL and the baseline model result in very similar accuracy of HSE arrival time prediction. Errors in the maximum speed of HSEs are also comparable.

[31] The physics-based models of the solar wind that form part of both WSA-ENLIL and CORHEL really begin to prove their worth in the morphology of HSEs. Figure 8 shows multiposed epoch plots of the correctly predicted HSEs, with black/red/green/blue showing observations/ baseline/WSA-ENLIL/CORHEL, using the time of maximum speed gradient as $t=0$. For clarity, error bars are not shown. In order to avoid averaging magnetic field components to zero, the magnetic field components of HSEs associated with heliospheric current sheet crossings in which the field goes from "inward-to-outward" polarities are flipped. This effectively means all HCS crossings are transformed to "outward-to-inward" polarity crossings. Figures $8 \mathrm{a}$ through $8 \mathrm{~h}$ are the parameters used to define metrics. We also show the the magnitude of the magnetic field components, labeled 1 through 3.

[32] Figure 8d shows the radial flow speed: it can be seen that on average both the peak solar wind speed and the duration of HSEs are very well matched by all the models. However, the speed of the CORHEL slow solar wind before and after the HSE is lower than observed (as reported in Table 1). As solar wind density is initiated by a parameterization of constant mass flux, it follows that CORHEL also over-predicts density before and after HSEs, as shown in Figure 8g.

[33] The MHD solar wind models both result in an underestimate in the magnetic flux in the heliosphere (Figure 8, labeled 1 and 2). The underestimate in flux may be related to the magnetograms and their processing [e.g., Ulrich, 1992], which in the context of assessing model prediction are treated as part of the model. The baseline model explicitly sets the field intensity at 1 AU to $5 \mathrm{nT}$ rather than using the open flux computed from the PFSS model, artificially matching the average field strength at 1 AU. Note that this method of adjusting $|\mathbf{B}|$ will not be able to match any time variation in heliospheric flux over the solar cycle [e.g., Owens and Crooker, 2006], which future models may be able to reproduce, allowing them to surpass the performance of the baseline model.

[34] CORHEL and WSA-ENLIL match the compression of the in-ecliptic magnetic field at the stream interface very well (Figures $8 \mathrm{a}$ and $8 \mathrm{~b}$ and labels 1 and 2). Similarly, $V_{Y}$, the in-ecliptic flow deflection (Figure 8e) is also as 


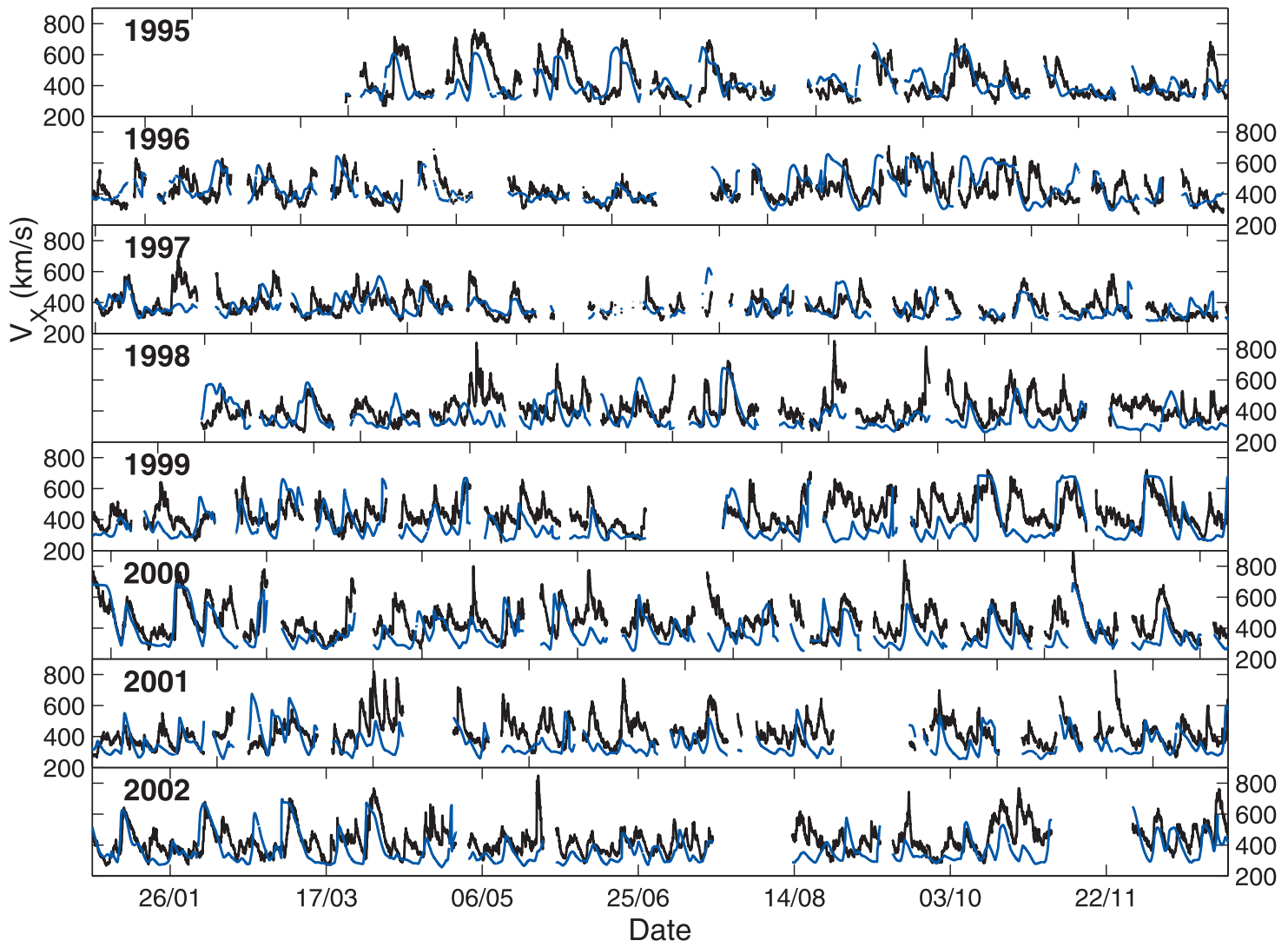

Figure 4. A comparison of the CORHEL prediction of the solar wind radial speed at L1 (blue) with that observed (black) for the years 1995-2002. Both time series are at 1-h resolution.

observed. The compression in the density is also well captured (Figure 8g). Ballistic propagation, as used in the baseline model, simply cannot capture these dynamic effects.

[35] Turning to the out-of-ecliptic magnetic fields, (labeled 3 in Figure 8) and flow deflections (not shown), WSA-ENLIL and CORHEL produce an enhancement in $\left|B_{Z}\right|$ and $\left|V_{Z}\right|$ in the HSE, as observed, but the background values of these parameters are far too low. The MHD models produce background $\left|B_{Z}\right|$ in GSE coordinates primarily due to the $7.25^{\circ}$ inclination between the helio- graphic equator and the ecliptic plane, however, this effect has a maximum value of $|B| \sin 7.25^{\circ} \sim 0.5 \mathrm{nT}$, compared to the $\sim 2 \mathrm{nT}$ observed. The additional shortfall may be due to the lack of small-scale turbulence in the MHD magnetic field and plasma flow, which causes fluctuations on time-scales much smaller than can be addressed with global MHD codes [e.g., Siscoe et al., 2004, and references therein]. In the actual components, not magnitudes, of the out-of-ecliptic field $\left(B_{Z}\right.$, Figure $\left.8 \mathrm{c}\right)$ and flow $\left(V_{Z}\right.$, Figure $\left.8 \mathrm{f}\right)$, both the observed and modeled enhancements are washed out by the averaging process, resulting in approx-

Table 1. Characteristic Properties of the Three Solar Wind Radial Speed Time Series for the Years 1995-2002

\begin{tabular}{|c|c|c|c|c|}
\hline & Observed & Baseline & WSA-ENLIL & CORHEL \\
\hline Number of data points & 59535 & 59535 & 59535 & 59535 \\
\hline Standard Deviation $\left|V_{X}\right|(\mathrm{km} / \mathrm{s})$ & 99.2 & 84.3 & 74.6 & 102 \\
\hline$r_{L}$ & - & 0.506 & 0.403 & 0.428 \\
\hline Skill (MSE) & - & - & -4.9 & -49.6 \\
\hline
\end{tabular}

${ }^{a}$ The top three rows are the number of data points, the mean value, and the standard deviation of the time series. The next three rows compare the model time series to the observations: the root mean square error and the linear correlation coefficients. Finally, the bottom row shows the skill of the model relative to the baseline. 

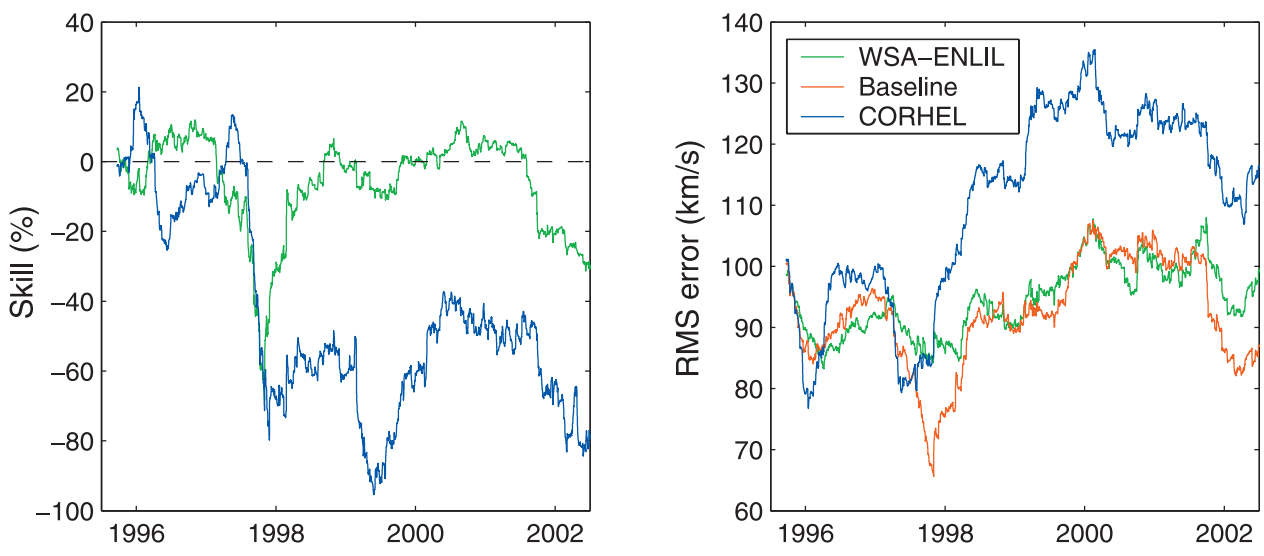

Time of 1-year bin centre

Figure 5. (left) The green (blue) plot shows the skill of the WSA-ENLIL (CORHEL) solar wind speed prediction relative to the baseline model for the years 1995-2002 as a function of time. A 365.25 day rolling window was used. (right) RMS error for the three model predictions: red, green, and blue for baseline, WSA-ENLIL, and CORHEL, respectively.

imately flat profiles. Finally, we note that the solar wind proton temperature is underestimated by all three coupled models. As temperature is currently a free parameter at the coronal-heliospheric interface, this may be fixed by further tuning. Despite the under-prediction, the temperature enhancement in the HSE is captured by WSAENLIL and CORHEL.

\subsection{Full Solar Wind Metrics}

[36] Table 4 lists the MSE and associated skill of CORHEL, WSA-ENLIL, and the baseline WSA model when predicting the various solar wind parameters over the whole 8-year period.
[37] First we consider the magnetic field: the magnitude of $B_{X}$ and $B_{Y}$ is overestimated in the baseline model. This is likely due to using the observed value of $|\mathbf{B}|$ at $1 \mathrm{AU}$, but imposing $B_{Z}=0$ (i.e., the observed value of $|\mathbf{B}|$ was decomposed into only two components, $B_{X}$ and $\left.B_{Y}\right)$ in the GSE coordinate system. The amount of open flux in the coronal model does not affect the baseline prediction of $|\mathbf{B}|$, thus adjusting $B_{X}$ and $B_{Y}$ to match observations in a climatological sense is trivial. Both WSA-ENLIL and CORHEL underestimate all three magnetic field components. As the magnetic field intensity is set by the amount of flux that opens in the WSA or MAS corona, observa-
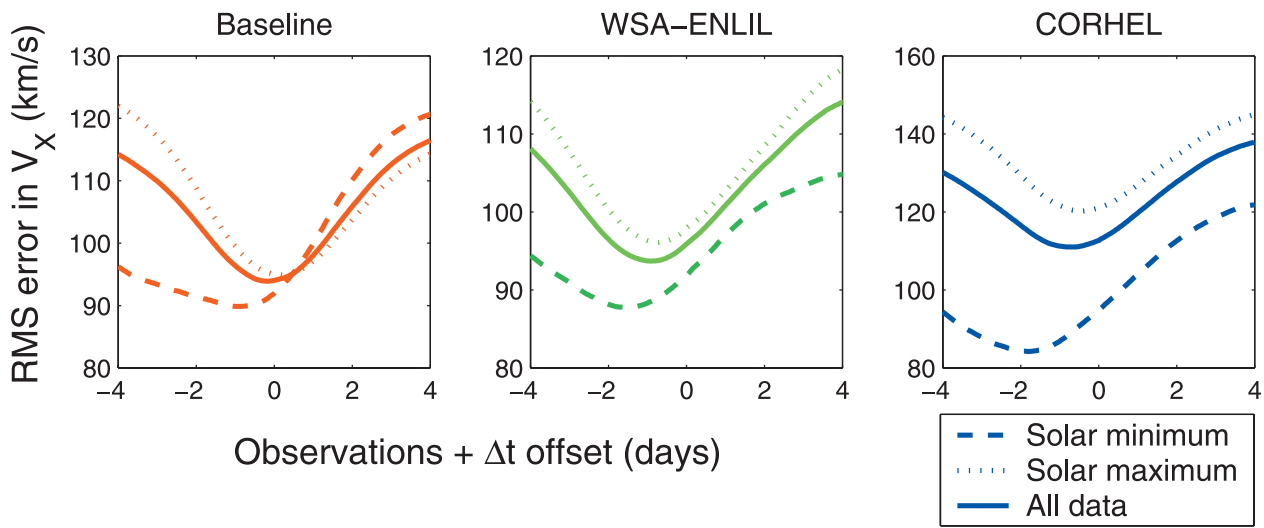

Figure 6. The systematic time offset in the model predictions of solar wind speed. The baseline model shows no significant systematic offset in the speed prediction. WSA-ENLIL and CORHEL, however, show an approximately 2-day early prediction of solar wind speed at solar minimum (dashed lines). This effect is less pronounced at solar maximum, when the RMS error is higher in general (dotted lines). 
Table 2. Contingency Tables Showing the Numbers of Hit, Miss, and False HSEs Using the Baseline, WSA-ENLIL, and CORHEL Predictions ${ }^{\mathrm{a}}$

\begin{tabular}{|c|c|c|c|c|c|c|c|}
\hline & & \multicolumn{2}{|c|}{ All Data Observed } & \multicolumn{2}{|c|}{$\begin{array}{c}\begin{array}{c}\text { Solar Minimum } \\
\text { Observed }\end{array} \\
\end{array}$} & \multicolumn{2}{|c|}{$\begin{array}{c}\text { Solar Maximum } \\
\text { Observed }\end{array}$} \\
\hline Model & $\begin{array}{l}\text { HSE } \\
\text { no HSE }\end{array}$ & $\begin{array}{l}\text { hit } \\
\text { miss }\end{array}$ & false alarm & & & & \\
\hline Baseline & $\begin{array}{l}\text { HSE } \\
\text { no HSE }\end{array}$ & $\begin{array}{l}138(138) \\
95(104)\end{array}$ & $26(27)$ & $\begin{array}{l}47(47) \\
29(33)\end{array}$ & $12(12)$ & $\begin{array}{l}91(91) \\
66(71)\end{array}$ & $14(15)$ \\
\hline WSA-ENLIL & $\begin{array}{l}\text { HSE } \\
\text { no HSE }\end{array}$ & $\begin{aligned} 90(90) \\
142(152)\end{aligned}$ & $17(18)$ & $\begin{array}{l}10(10) \\
64(70)\end{array}$ & $\begin{array}{c}3(3) \\
-\end{array}$ & $\begin{array}{l}80(80) \\
78(82)\end{array}$ & $14(15)$ \\
\hline
\end{tabular}

${ }^{a}$ The values in parentheses represent the statistics before data gap considerations are made. We do not consider true negative predictions.

tions can only be matched by forcing the coronal solution to open more flux.

[38] The skill of the radial speed predictions were discussed in detail in the previous section. The nonradial solar wind speeds highlight a potential pitfall with the interpretation of point-by-point metrics such as MSE and correlation: Despite CORHEL (and to a lesser extent, WSA-ENLIL) capturing both the direction and magnitude of the $V_{Y}$ deflections very well, the baseline model has a lower MSE, even though it produces no $V_{Y}$ deflections at all (as shown by both Table 4 and Figure 8 ). This is due to timing errors in the deflections. A similar effect is present for $B_{X}$ and $B_{Y}$. It could be argued that the "flawed" CORHEL predictions (in terms of MSE and correlation coefficients) are more desirable for both storm prediction
Baseline
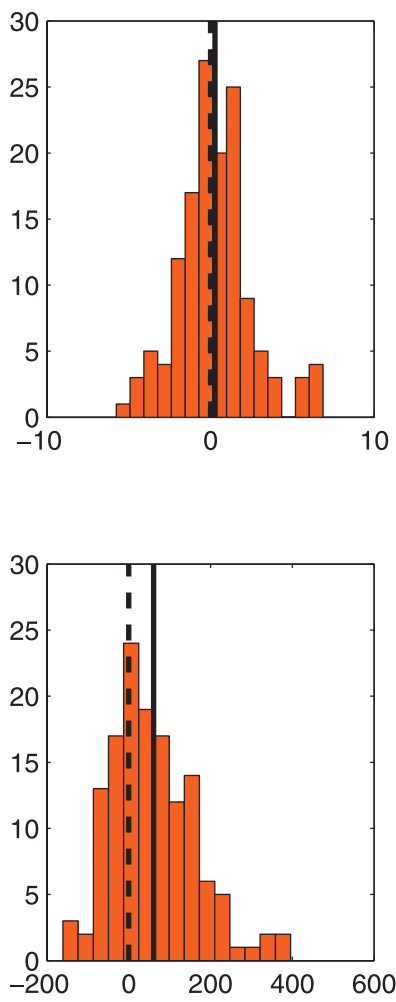

WSA-ENLIL

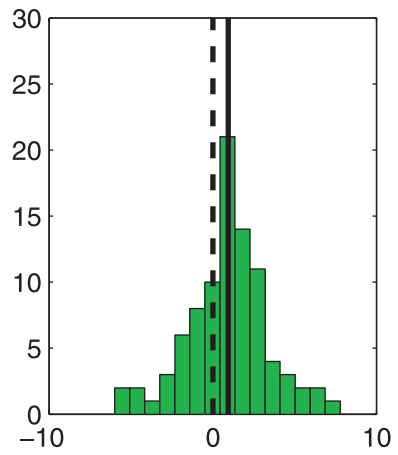

dT (observed-model) (days)

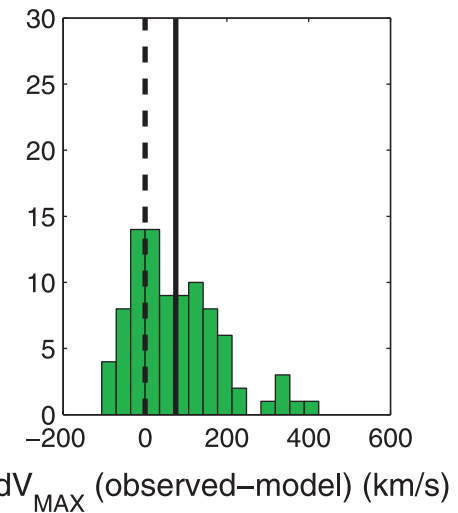

CORHEL

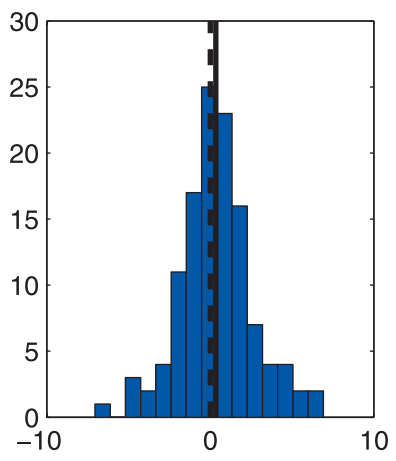

10

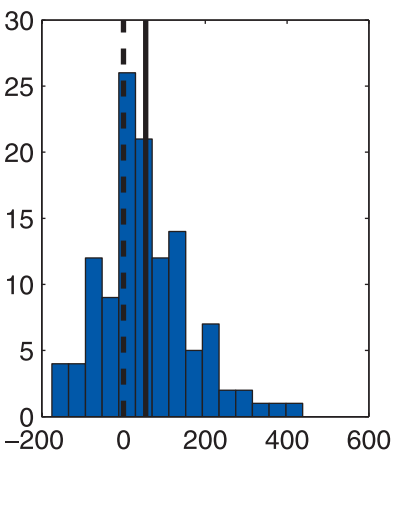

Figure 7. The distributions of the errors in (top) the arrival time and (bottom) maximum speed of HSEs predicted by three models. The solid vertical lines show the mean of the distributions. 
Table 3. Accuracy of the Model-Predicted Arrival Times and Maximum Speeds of HSEs ${ }^{\mathrm{a}}$

\begin{tabular}{|c|c|c|c|c|}
\hline & $\langle d T\rangle$ (days) & $\langle|d T|\rangle$ (days) & $\langle d V\rangle(\mathrm{km} / \mathrm{s})$ & $\langle|d V|\rangle(\mathrm{km} / \mathrm{s})$ \\
\hline \multicolumn{5}{|c|}{ All Data } \\
\hline WSA-ENLIL & 0.94 & 2.08 & 75.1 & 97.2 \\
\hline \multicolumn{5}{|c|}{ Solar Minimum (1995-1997) } \\
\hline Baseline & 0.45 & 1.86 & 35.3 & 78.9 \\
\hline WSA-ENLIL & 2.69 & 2.69 & 69.2 & 106.7 \\
\hline \multicolumn{5}{|c|}{ Solar Maximum (1998-2002) } \\
\hline Baseline & 0.20 & 1.58 & 74.3 & 96.5 \\
\hline WSA-ENLIL & 0.72 & 2.00 & 75.9 & 96.0 \\
\hline CORHEL & 0.07 & 1.60 & 62.3 & 100.1 \\
\hline
\end{tabular}

${ }^{a}$ Here $d T$ is observated - model arrival time. $\langle d T\rangle$ and $\langle d V\rangle$ quantify any systematic offsets, while $\langle|d T|\rangle$ and $\langle|d V|\rangle$ represent the average error.

and driving geospace simulations than those of the baseline model.

[39] Figure 8g shows how the ENLIL model of the solar wind (employed in both WSA-ENLIL and CORHEL) accurately captures the density enhancement that occurs at a solar wind stream interface. However, both WSA-ENLIL and CORHEL have negative skill scores for solar wind density, despite the baseline model producing no density enhancement at a stream interface. This is again the result of errors in the timing of the stream interface, as was seen for $V_{Y}, V_{Z}, B_{X}$ and $B_{Y}$, though a systematic offset to the average density also contributes. Finally, we note once more the underestimate of the temperature. This is due to ENLIL being intialised with too low a temperature. As

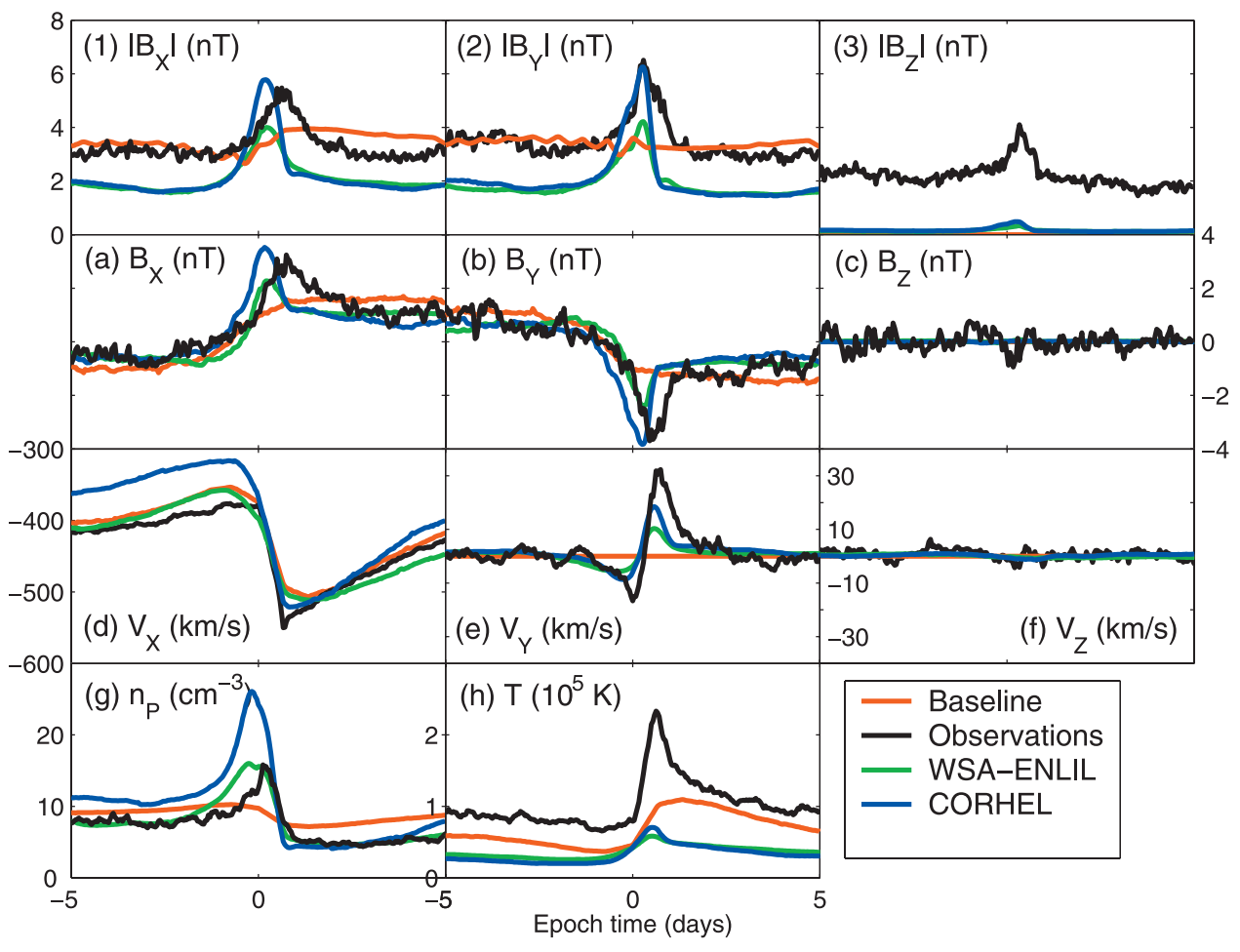

Figure 8. Multiposed epoch plots of the correctly predicted HSEs, with black/red/green/blue showing observations/baseline/WSA-ENLIL/CORHEL, using the time of maximum speed gradient as $t=0$. All HCS crossings have been transformed to "outward-to-inward" polarities to avoid averaging the magnetic field components to zero. (a) through (h) The parameters used to define metrics. We also show the the magnitude of the magnetic field components, labeled 1 through 3. 
Table 4. Statistics of the Observed, Baseline, WSA-ENLIL, and CORHEL Time Series

\begin{tabular}{|c|c|c|c|c|c|c|c|c|}
\hline & $B_{X}(\mathrm{nT})$ & $B_{Y}(\mathrm{nT})$ & $B_{Z}(\mathrm{nT})$ & $V_{X}(\mathrm{~km} / \mathrm{s})$ & $V_{Y}(\mathrm{~km} / \mathrm{s})$ & $V_{Z}(\mathrm{~km} / \mathrm{s})$ & $n_{P}\left(\mathrm{~cm}^{-3}\right)$ & $T_{P}\left(10^{5} \mathrm{~K}\right)$ \\
\hline Number of points used & 61,029 & 61,029 & 61,029 & 59,535 & 59,535 & 59,535 & 55,985 & 55,984 \\
\hline \multicolumn{9}{|c|}{ Observations } \\
\hline$<|X|>$ & 3.24 & 3.48 & 2.17 & 434.3 & 16.6 & 14.7 & 7.47 & 1.01 \\
\hline STD & 3.90 & 4.43 & 3.22 & 99.2 & 22.9 & 19.3 & 5.73 & 0.777 \\
\hline \multicolumn{9}{|c|}{ Baseline } \\
\hline STD & 3.50 & 3.50 & 0 & 84.36 & 0 & 0 & 1.70 & 0.407 \\
\hline RMS & 3.66 & 4.33 & 3.22 & 94.9 & 22.9 & 19.7 & 5.83 & 0.826 \\
\hline$r_{L}$ & 0.52 & 0.42 & N/A & 0.506 & N/A & N/A & 0.199 & 0.356 \\
\hline \multicolumn{9}{|c|}{ WSA-ENLIL } \\
\hline$<X>$ & -0.059 & 0.021 & -0.0033 & -430.0 & 0.52 & -0.037 & 7.57 & 0.370 \\
\hline$<|X|>$ & 2.14 & 1.92 & 0.158 & 430.9 & 2.82 & 2.04 & 7.57 & 0.370 \\
\hline \multicolumn{9}{|c|}{ CORHEL } \\
\hline$<X>$ & -0.119 & 0.066 & -0.0084 & -389.7 & 1.33 & -0.073 & 9.84 & 0.316 \\
\hline$<|X|>$ & 2.18 & 2.06 & 0.168 & 389.7 & 4.25 & 3.22 & 9.84 & 0.316 \\
\hline STD & 2.49 & 2.40 & 0.218 & 102.0 & 6.35 & 4.29 & 6.77 & 0.180 \\
\hline RMS & 3.68 & 4.31 & 3.22 & 116 & 23.4 & 19.9 & 8.61 & 1.02 \\
\hline$r_{L}$ & 0.41 & 0.32 & 0.018 & 0.428 & 0.0661 & 0.041 & 0.130 & 0.319 \\
\hline Skill & -1.10 & 0.92 & 0 & -50.7 & -4.41 & -2.04 & -118 & -52.5 \\
\hline
\end{tabular}

temperature is currently a free parameter at the coronaheliosphere coupling interface, this can be improved with further parameterization of the model coupling.

\section{Conclusions}

[40] We have performed a systematic comparison of 8 years of solar wind observations with predictions made by the empirical baseline model, the WSA-ENLIL empirical/physics-based hybrid model and the full MHD CORHEL model. The radial solar wind speed is the most critical parameter in solar wind models, as it orders all of the other solar wind properties. Over the whole 8-year period, it was found that the skill of the WSA-ENLIL prediction of $V_{X}$ was comparable to that of the baseline prediction. However, the lack of variability in the WSAENLIL solar wind speed during 1995 and 1996 suggests that further tuning of the coupling interface between the WSA coronal model and the ENLIL heliospheric model is required. As the solar wind speed at $5 R_{S}$ predicted by the WSA corona was originally tuned to match observations via ballistic propagation to $1 \mathrm{AU}$, this is to be expected. Work is currently underway to better calibrate the WSA solar wind speeds for use in ENLIL.

[41] The skill of the CORHEL prediction of $V_{X}$ is around zero (i.e., the errors in the predictions of CORHEL and the baseline were comparable) for the years 1995-1997. However, there is a drop in skill in 1998, strongly associated with an underestimate in the mean solar wind speed at this time. In this version of CORHEL, a polytropic energy equation is used, which requires an empirical method for specifying a realistic solar wind speed as the lower boundary condition of the heliospheric model. The systematic underestimate of the solar wind speed by CORHEL may occur because the model parameters in equation (5) have not been tuned as well as the WSA model, where far more effort has been expended on comparing with solar wind speed thus far. MHD models with a more realistic energy equation [Lionello et al., 2001; Lionello et al., 2007] may be able to provide more accurate solar wind speed estimates in a more self-consistent manner. We also note the systematic time offset in the CORHEL and WSA-ENLIL time series. This is likely due to the propagation time between the photosphere and corona-heliosphere boundary not being accounted for as the coronal models do not explicitly include solar rotation. In future iterations of the models such effects may be incorporated, but as an interim fix, the introduction of a constant time shift to the model time series might improve their predictive capabilities. Without further investigation, however, it is unclear whether the time delay derived from the RMS or the event-based analysis should be used. Furthermore, asymmetric velocity features (such as a preponderance of sharp rises and slow decays) may skew the results.

[42] An events-based approach was also applied to the solar wind speed predictions, with high-speed enhancements (HSEs) selected as the significant features in the solar wind time series [Owens et al., 2005]. The ratio of hit/ miss/false HSEs suggests the CORHEL reconstruction of 
the solar wind is of similar accuracy to that of the baseline. The hit rate of WSA-ENLIL is significantly lower, due the lack of variability in the predicted solar wind speed around solar minimum. This problem can likely be addressed by a reparameterization of the WSA-ENLIL coupling interface.

[43] Other solar wind parameters, such as magnetic field components, nonradial flows and density, are ordered by $V_{X}$, and thus the MSE is severely influenced by timing errors. Indeed, the $B_{X}$ and $B_{Y}$ enhancements associated with HSEs are very well captured by CORHEL and WSAENLIL, but errors in timing result in a lower MSE for the baseline prediction of no enhancement at all. Solar wind density shows a similar effect, though errors are further amplified by the general overestimation of density. As the solar wind density is scaled by mass conservation, underestimating of the speed will necessarily overestimate the density.

[44] In summary, the empirical baseline scheme is relatively mature and, as such, has undergone a great deal of tuning and testing. As a result, it currently produces the "best" prediction of solar wind parameters in near-Earth space, at least in terms of mean-square-error. The newer physics-based approaches still require some further parameterization but show a great deal of promise as powerful predictive tools, even in the low-resolution modes explored in this study. With continual development, the physics-based approach has the potential to surpass the predictive capability of empirical schemes, particularly when it comes to the integration of transient structures, the drivers of major space weather disturbances.

[45] Acknowledgments. This research was supported by CISM which is funded by the National Science Foundation STC program under agreement ATM-012950.

\section{References}

Altschuler, M. A., and G. Newkirk Jr. (1969), Magnetic fields and the structure of the solar corona, Sol. Phys., 9, 131-149.

Arge, C. N., and V. J. Pizzo (2000), Improvement in the prediction of solar wind conditions using near-real time solar magnetic field updates, J. Geophys. Res., 105, 10,465-10,479.

Arge, C. N., D. Odstrcil, V. J. Pizzo, and L. R. Mayer (2003), Improved method for specifying solar wind speed near the Sun, in Solar Wind Ten, edited by M. Velli, R. Bruno, and F. Malara, AIP Conf. Proc., 679, $190-193$.

Arge, C. N., J. G. Luhmann, D. Odstrcil, C. J. Scrijver, and Y. Li (2004), Stream structure and coronal sources of the solar wind during the May 12th, 1997 CME, J. Atmos. Sol. Terr. Phys., 66, 1295-1310.

de Toma, G., C. N. Arge, and P. Riley (2005), Observed and modeled coronal holes, in "Connecting Sun and Heliosphere": Proceedings of Solar Wind 11/SOHO 16, edited by B. Fleck and T. H. Zurbuchen, Eur. Space Agency Spec. Publ., ESA SP-592, 609.

Feynman, J., and S. B. Gabriel (2000), On space weather consequences and predictions, J. Geophys. Res., 105, 10,543-10,564.

Harvey, K. L., and F. Recely (2002), Polar coronal holes during cycles 22 and 23, Sol. Phys., 211, 31-52.

Linker, J., et al. (1999), Magnetohydrodynamic modeling of the solar corona during whole sun month, J. Geophys. Res., 104, 9809-9830.
Linker, J. A., and Z. Mikić, (1997), Extending coronal models to earth orbit, in Coronal Mass Ejections, Geophys. Monogr. Ser., vol. 99, edited by N. Crooker, J. Joselyn, and J. Feynman, pp. 269-278, AGU, Washington, D. C.

Lionello, R., Z. Mikić, and J. A. Linker (1999), Stability of algorithms for waves with large flows, J. Comput. Phys., 152, 346-358.

Lionello, R., J. A. Linker, and Z. Mikic (2001), Including the transition region in models of the large-scale solar corona, Astrophys. J., 546, $542-551$.

Lionello, R., J. A. Linker, and Z. Mikić (2007), The multispectral emission of the Sun during August 1996, Am. Astron. Soc. Meet. Abstr., 210, 91.08 .

Lopez, R. E. (1987), Solar-cycle invariance in the solar wind proton temperature relationships, J. Geophys. Res., 92, 11,189-11,194.

Luhmann, J. G., S. C. Soloman, J. A. Linker, J. G. Lyon, Z. Mikic, D. Odstrcil, W. Wang, and M. Wiltberger (2004), Coupled model simulation of a Sun-to-Earth space weather event, J. Atmos. Sol. Terr. Phys., 66, 1243-1256.

Mikic, Z., J. A. Linker, D. D. Schnack, R. Lionello, and A. Tarditi (1999), Magnetohydrodynamic modeling of the global solar corona, Phys. Plasma, 6, 2217-2224.

Odstrcil, D. (2003), Modeling 3-D solar wind structures, Adv. Space Res., 32, 497-506.

Odstrcil, D., V. Pizzo, J. A. Linker, P. Riley, R. Lionello, and Z. Mikic (2004), Initial coupling of coronal and heliospheric numerical magnetohydrodynamic codes, J. Atmos. Sol. Terr. Phys., 66, 1311-1320.

Owens, M. J., and N. U. Crooker (2006), Coronal mass ejections and magnetic flux buildup in the heliosphere, J. Geophys. Res., 111, A10104, doi:10.1029/2006JA011641.

Owens, M. J., C. N. Arge, H. E. Spence, and A. Pembroke (2005), An event-based approach to validating solar wind speed predictions: High speed enhancements in the Wang-Sheeley-Arge model, J. Geophys. Res., 110, A12105, doi:10.1029/2005JA011343.

Richardson, I. G., H. V. Cane, and E. W. Cliver (2002), Sources of geomagnetic activity during nearly three solar cycles (1972-2000), J. Geophys. Res., 107(A8), 1187, doi:10.1029/2001JA000504.

Riley, P., J. A. Linker, and Z. Mikic (2001), An empirically-driven global MHD model of the solar corona and inner heliosphere, J. Geophys. Res., 106, 15,889-15,902.

Schatten, K. H. (1971), Current sheet magnetic model for the solar corona, Cosmic Electrodyn., 2, 232-245.

Schatten, K. H., J. M. Wilcox, and N. F. Ness (1969), A model of interplanetary and coronal magnetic fields, Sol. Phys., 9, 442-455.

Siscoe, G., D. Baker, R. Weigel, J. Hughes, and H. Spence (2004), Roles of empirical modeling within CISM, J. Atmos. Sol. Terr. Phys., 66, 1469-1480.

Spence, H., D. Baker, A. Burns, T. Guild, C.-L. Huang, G. Siscoe, and R. Weigel (2004), Center for integrated space weather modeling metrics plan and initial model validation results, J. Atmos. Sol. Terr. Phys., 66, 1491-1498.

Tsurutani, B. T., W. D. Gonzalez, F. Tang, S. I. Akasofu, and E. J. Smith (1988), Origin of the interplanetary southward magnetic fields responsible for the major magnetic storms near solar maximum (1978-1979), J. Geophys. Res., 93, 8519-8531.

Ulrich, R. K. (1992), Analysis of magnetic fluxtubes on the solar surface from observations at Mt. Wilson of A5250; A5233, in Cool Stars, Stellar Systems, and the Sun: Seventh Cambridge Workshop, Conf. Ser., vol. 26, edited by M. S. Giampapa and J. A. Bookbinder, p. 265, Astron. Soc. of the Pac., San Fransisco, Calif.

C. N. Arge, Space Vehicles Directorate, Air Force Research Laboratory, Kirtland Air Force Base, NM 87117, USA.

W. J. Hughes, S. McGregor, M. J. Owens, J. M. Quinn, and H. E. Spence, Center for Space Physics, Boston University, Boston, MA 02215, USA. (mjowens@bu.edu)

J. Linker and P. Riley, Science Applications International Corporation, 10260 Campus Point Drive, San Diego, CA 92121, USA.

D. Odstrcil, Cooperative Institute for Research in Environmental Sciences, University of Colorado, Boulder, CO 80309, USA. 